(C) 1998 IEEE. Personal use of this material is permitted. Permission from IEEE must be obtained for all other uses, in any current or future media, including reprinting/republishing this material for advertising or promotional purposes, creating new collective works, for resale or redistribution to servers or lists, or reuse of any copyrighted component of this work in other works. 


\title{
PARAMETER OPTIMIZATION AND SIMULATED PERFORMANCE OF A DVB-T DIGITAL TELEVISION BROADCASTING SYSTEM
}

\author{
Ana Garcia Armada, Beatriz Bardón, Miguel Calvo \\ Grupo de Radiación, Dpto. SSR \\ Polytechnic University of Madrid (SPAIN) \\ Tel: +3413367360 Fax: +3415432002 \\ e-mail: ana@gr.ssr.upm.es
}

\begin{abstract}
A prototype of a Digital Television Terrestrial Broadcasting System has been implemented according to the European IVB-T standard. The first step in the construction of this prototype involved parameter optimization via simulation.

This paper presents the optimization process of the system parameters and the final achieved BER performance. Important design aspects such as interleaving, convolutional puncturing codes, Viterbi decoding, pilot-besed channel correction and optimal pilot power are considered.

The DVB-T standard offers some suggested values of the $\mathrm{C} / \mathrm{N}$ ratio neteded to achieve the desired $\mathrm{BER}$ requirements in the receiver. However, they have been obtained assuming ideal channel correction and synchronization.
\end{abstract}

Simulation results are provided here in non-ideal conditions.

\section{INTRODUCTION}

In the European DVB Project (Digital Video Broadcasting), founded by initiative of more than 190 organizations, the standards for digital television broadcasting have been specified for the different transmission media: satellite (DVB-S), cable (DVB-C) and terrestrial (DVB-T) [1]. The later is a result of the RACE Project dT'Tb (Digital Television for Terrestrial Broadcasting), which has covered all the aspects of digital television terrestrial broadcasting in Europe, with the work of 30 partners from 10 different countries [2].

The system specified within the dTTb Project is very complex [1,2]. Taking the input signal coming from an MPEG-2 encoder, all the elements for its correct transmission and reception are defined, adapting the signal to the terrestrial channel. With this aim, a combined encoding and modulation process [3] is applied to the data source, with different coding rates [4] (which imply different bit rates), according to the channel characteristics and the expected quality at the receiver. Besides, different services, depending on the quality of the received TV signal (standard, enhanced and high definition) and several configurations (by combination of these services) are defined.
In order to build a prototype for such a system, we have developed a methodology to allow the use of software design tools to implement in a rapid and efficient way communications systems prototypes using digital signal processors (DSPs) [5]. The first step in the implementation process is the optimization of every parameter to achieve the system BER requirements.

In particular, the influence on the BER performance of the following elements has been studied:

- interleaving

- convolutional punctured codes and Viterbi decoding

- pilot-based channel correction

- optimum power of pilot sub-carriers

In the next section, the transmitter and receiver are described. Following, the optimized system parameters and the final achieved BER performance are presented. These results are compared with the DVB-T standard suggested performance.

\section{DESCRIPTION OF TRANSMITTER AND RECEIVER ELEMENTS}

In this section, the transmitter and receiver of the prototype, which implement the main functions defined in the DVB-T standard [1], are described.

The elements of the transmitter, whose block diagram is shown in Figure 1, are the following:

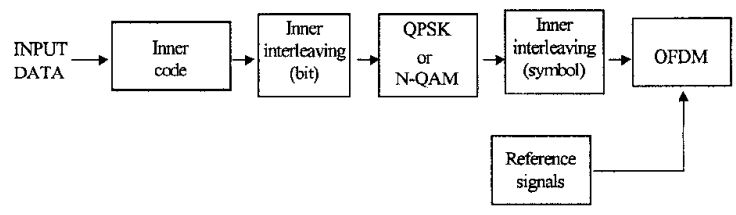

Figure 1 Transmitter block diagram

\subsection{Inner coding}

Inner coding is based on $1 / 2$ convolutional code, with constraint length 7 and generator polynomials $171_{\text {Oct }}$, $133_{\text {Oct }}$. This mother code is used to produce several punctured codes with different coding rates [4]. 
Table 1 indicates the bits that must be punctured in order to construct the different codes with $x 1, \times 2, \times 3, \ldots$ the output bits of mother code.

Table 1 Puncturing patterns

\begin{tabular}{|c|c|c|}
\hline rate & pattern & transmitted sequence \\
\hline $1 / 2$ & 11 & $\times 1 \times 2$ \\
$2 / 3$ & 1101 & $\times 1 \times 2 \times 4$ \\
$3 / 4$ & 110110 & $\times 1 \times 2 \times 4 \times 5$ \\
$5 / 6$ & 1101100110 & $\times 1 \times 2 \times 4 \times 5 \times 8 \times 9$ \\
$7 / 8$ & 11010101100110 & $\times 1 \times 2 \times 4 \times 6 \times 8 \times 9 \times 12 \times 13$ \\
\hline
\end{tabular}

\subsection{Inner interleaving}

The DVB-T standard specifies a combination of bit and symbol inner interleaving: a first block interleaving is applied to the bits with 126-bit blocks and a second block interleaving is applied to the digital modulation symbols. The block length of this second interleaving is equal to the number of information sub-carriers in the OFDM frame (1512 in 2K-OFDM and 6048 in 8KOFDM).

\subsection{Digital modulation}

Digital modulation is based on QPSK, 16-QAM or 64QAM. It is a multi-resolution modulation which allows one to design a hierarchical error protection scheme.

The constellation is divided in clusters that are further divided in sub-clusters. The higher priority bits (HP) are mapped to the clusters and the lower priority ones to the sub-clusters (MP) or constellation points (LP).

The euclidean distances between clusters $d_{E I}$, subclusters $d_{E 2}$ and points $d_{E 3}$ can be changed in order to obtain different degrees of protection. Figure 2 shows an example for 16-QAM with $\alpha_{1}=d_{E I} / d_{E 2}$.

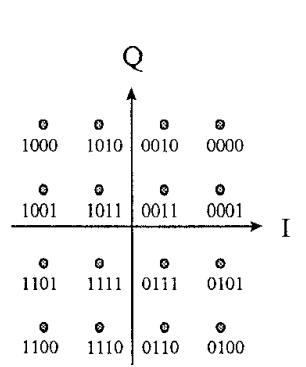

(a) 16-QAM, $\alpha_{1}=1$

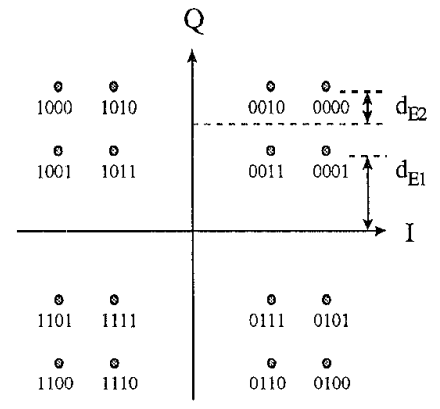

(b) 16-QAM, $\alpha_{1}=2$
Figure 2 Multi-resolution 16-QAM

Besides modulation and coding are combined following a multi-level coding scheme [3].

\subsection{Orthogonal Frequency Division Multiplexing (OFDM)}

OFDM is one of the best alternatives to alleviate the multipath effects in mobile communications [6]. For reception in built-up areas with delay spreads of some microseconds, the channel is highly frequency selective and sophisticated equalization techniques are required to achieve high bit rate transmissions. However, a combination of OFDM and coding associated with interleaving in the frequency domain (COFDM) can take advantage from the diversity associated to multipath [7]. Besides, multi-carrier systems have been proved to be more computationally efficient than single-carrier systems with equalization under some multipath channel conditions [8].

In an OFDM system the spectrum associated to each elemental data is a small portion of the total bandwidth, which is divided in $N$ sub-channels. Each of them is modulated with one symbol and they are all multiplexed in frequency.

If $T$ represents the OFDM symbol duration, that is: $T=\frac{N}{B}$, the $N$ carriers are placed in the frequencies:

$$
f k=f_{o}+k / T, \quad k=1,2, \ldots, N-1
$$

A higher spectral efficiency is achieved because the different sub-channels are allowed to overlap.

Orthogonality between them simplifies the separation process in the receiver, allowing the use of the Discrete Fourier Transform [9].

The complex envelope of the transmitted OFDM signal, sampled with sampling frequency $f_{S}=\frac{1}{B}$, is:

$$
s(n)=\sum_{m=-\infty}^{\infty} \sum_{n=N_{g}}^{N-1} \sum_{k=0}^{N-1} s_{m, k} \cdot e^{j \frac{2 \pi}{N} k n} \prod\left(n-m\left(N+N_{g}\right)\right)
$$

This means that every OFDM symbol has $N+N_{g}$ values: the $N$ obtained by an Inverse Discrete Fourier Transform (IDFT) plus an added Time Guard of $N_{g}$. This Time Guard consists of the last $N_{g}$ samples of the IDFT which are added at the beginning of each symbol in order to cope with the ISI caused by multipath delay spread.

$\Pi(n)$ is a rectangular pulse of duration $N+N_{g}$ samples.

Two frequency guards are also inserted, leaving some sub-carriers without modulation at the lower and upper sides of the spectrum, as a protection against adjacent channel interference $(\mathrm{ACI})$.

Besides some sub-carriers (called pilots) are transmitted with a known amplitude in order to provide a means of channel estimation and correction. 


\subsection{Transmitted signal}

The COFDM signal is transmitted in QAM (quadrature amplitude modulation) with a first intermediate frequency $I F_{1}=4.57 \mathrm{MHz}$, that is, half the sampling frequency of the complex lowpass signal. The complex signal is interpolated by a factor of two before translation to the II frequency. Its spectrum is shown in

Figure 3.

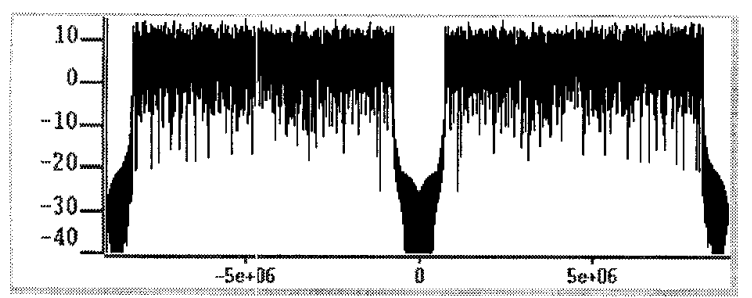

Figure 3 Spectrum of QAM transmitted signal

The transmitter blcck diagram is shown in Figure 4.

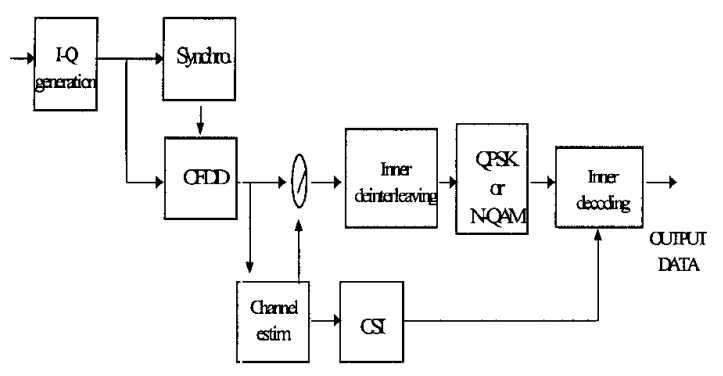

Figure 4 Receiver block diagram

Its main elements are the following:

\subsection{I-Q generation}

Three means of generating the I-Q components from the real received signal have been investigated:

- Traditional I-Q generation

- Frequency conversion using a sampling frequency equal to $4 I F_{I}$ as suggested in $\mathrm{dTTb}$

- Use of the Hilbert transform

The traditional method exhibits some disadvantages, such as sensitivity to frequency or phase mismatch between transmitter and receiver local oscillators, that are overcome by the two proposed digital methods.

While both digital schemes offer the same performance in AWGN, the method based on the Hilbert transform performs better in situations of frequency instability or timing jitter.

\subsection{OFDM frame synchronization and Orthogonal Frequency Division Demultiplexing}

Three synchronization operations are performed in this system:

$$
\begin{aligned}
& \text { - Identification of the number of sub-carriers } \\
& \text { - Time Guard estimation } \\
& \text { - FFT window adjustment }
\end{aligned}
$$

They are all accomplished by using the following correlation function [10]:

$$
\operatorname{corr}(n)=\sum_{k=n-N_{I}+1}^{n} x(k) \cdot x^{*}(k-N)
$$

The Orthogonal Frequency Division Demultiplexing (OFDD) process is implemented by means of a Fast Fourier Transform [9].

\subsection{Channel estimation and correction}

The channel effects in OFDM can be corrected by interpolating in time and frequency the DFT of the channel impulse response with the aid of the pilot subcarriers inserted in the OFDM frame. This process is shown in Figure 5. More details can be found in [11].

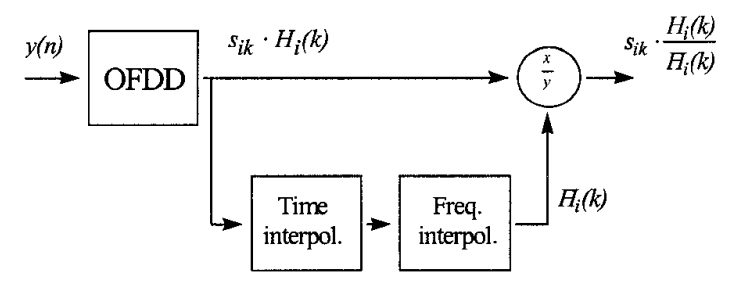

Figure 5 Channel correction by pilot-based interpolation

\subsection{Digital demodulation and inner decoding}

The use of multi-level coding allows one to implement the demodulation-decoding process in a sub-optimal way by means of multistage decoding [12], reducing the complexity of the optimal maximum likelihood decoder. This technique consists in decoding in a sequential fashion the different levels of priority, using the information of the already decoded HP bits for the decoding of the MP bits, and both the HP and MP information for the decoding of the LP bits.

The decoding of each bit stream is accomplished by using the Viterbi algorithm with a previous insertion of dummy bits for the codes generated by puncturing.

The inclusion of channel state information (CSI) in the Viterbi metrics has been considered. 


\section{PARAMETER OPTIMIZATION}

The main parameters related to the described elements have been optimized so as to achieve the system BER requirements. Some of them are discussed here.

\subsection{Interleaving}

As mentioned in the preceding section, the DVB-T standard specifies a combination of bit and symbol inner interleaving. Such an interleaving requires memory and causes delay in the transmitter and receiver.

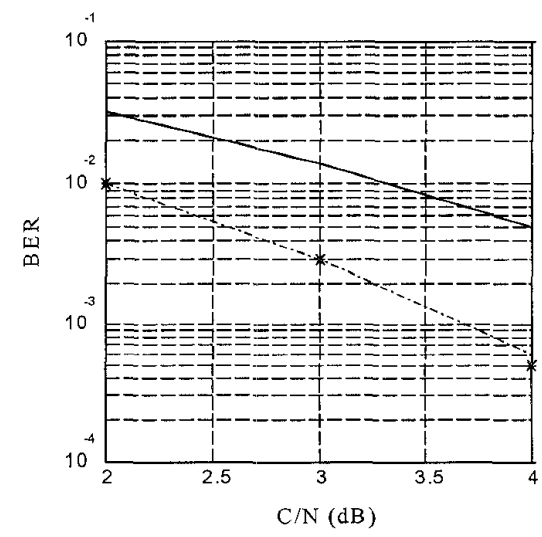

Figure 6 BER in a 2K-OFDM system with QPSK: no interleaving (-), symbol interleaving (--), bit and symbol interleaving $(*)$

It can be questioned if such a complex interleaving is needed or only the symbol interleaving, which corresponds to the COFDM concept, would be enough. Simulations have been run to answer this question when the OFDM sub-carriers are modulated in QPSK and 16-QAM. Results are shown in Figures 6 and 7.

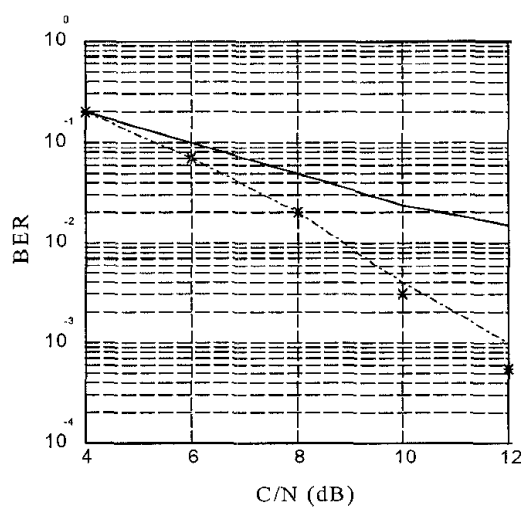

Figure 7 BER in a $2 \mathrm{~K}-\mathrm{OFDM}$ system with 16 QAM: no interleaving (-), symbol interleaving (--), bit and symbol interleaving $(*)$
In these simulations the OFDM signal has been transmitted through a multi-path channel named F1 whose definition can be found in [11].

It can be noticed that a combination of bit and symbol interleaving offers a negligible gain when the digital modulation of the sub-carriers is QPSK. However, when using a higher level modulation such as 16QAM, a combined interleaving can be necessary to achieve the BER requirements.

\subsection{Inner decoding}

Two main parameters have to be adjusted in relation to the inner decoder: the Viterbi decoding path length and the number of quantization levels. Figure 8 shows the bit error rate for different values of the decoding path length when using a $3 / 4$ rate punctured code. As it can be seen, a value of 30 corresponds to an optimum trade-off between memory and performance.

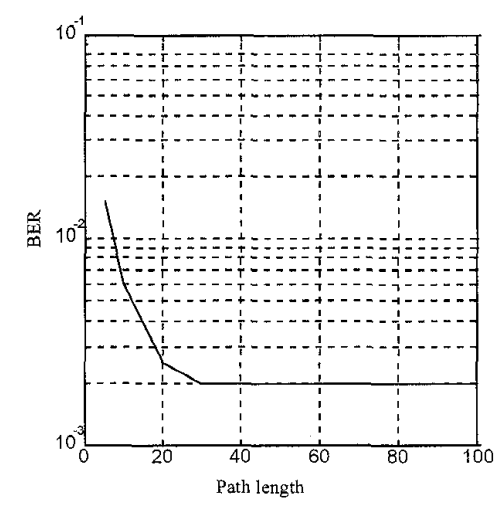

Figure 8 BER of $3 / 4$ rate punctured code vs. decoding path length for $\mathrm{C} / \mathrm{N}=3 \mathrm{~dB}$

Figure 9 shows the performance of the same code when two different numbers of quantization levels are used: the upper curve corresponds to an 8-level quantization while the lower curve has been obtained with 32 levels.

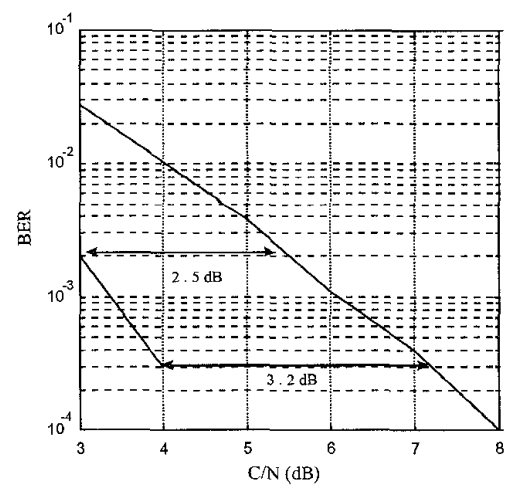

Figure 9 BER of $3 / 4$ rate punctured code vs. $C / N$ 
Although the use of 32 levels of quantization instead of 8 for the mother $1 / 2$ convolutional code leads to a poor gain $(0.25 \mathrm{~dB})$, we can see that this gain is much bigger when using punctured codes.

\subsection{Pilot-based channel correction}

Figure 10 shows the symbol error rate (SER) obtained in an OFDM system after the F1 channel [11] when pilot-based channel correction is used [11]. For $2 \mathrm{~K}$ and $8 \mathrm{~K}$ systems, channel correction leads to a better performance, but this only occurs when the $C / N$ ratio exceeds some value (around $10 \mathrm{~dB}$ ).

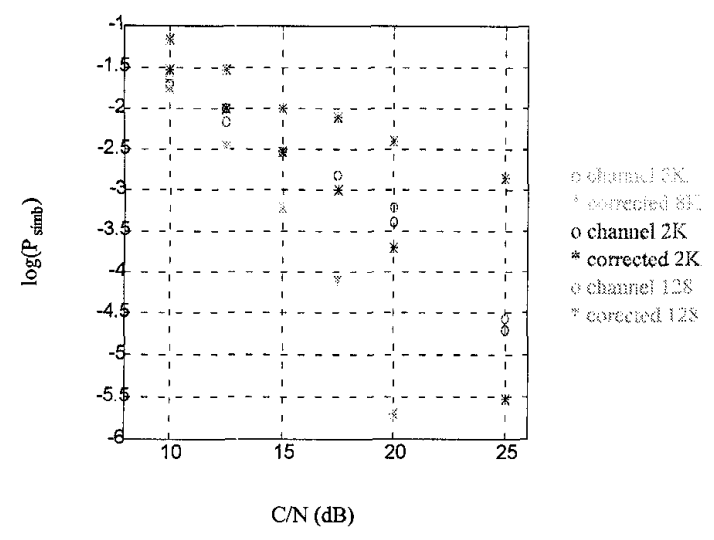

Figure 10 SER versus $\mathrm{C} / \mathrm{N}$ with and without channel correction

Since the system working point can be below this value, some simulations have been run on order to check in what situation a correction scheme is needed.

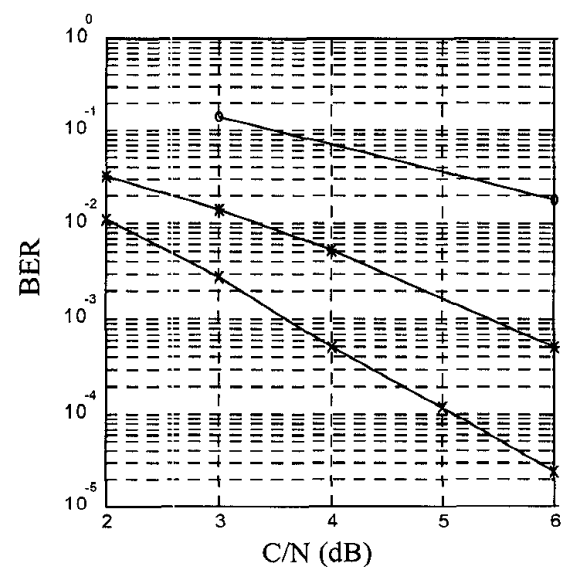

Figure 11 BER of QPSK-OFDM (2K) after F1 channel

The bit error rate (BER) for a $2 \mathrm{~K}$ system using QPSK can be seen in Figure 11. Three cases have been considered: channel correction without interleaving (o), no channel correction nor interleaving $(*)$, no channel correction and with symbol interleaving $(\mathrm{x})$.

It can be noticed that for the considered system and $C / N$ values, the correction of the channel effects leads to a worse performance, since the channel estimation is corrupted by noise.

However, when using 16-QAM instead of QPSK, the working point corresponds to higher $C / N$ values and the channel correction scheme provides better performances. This fact can be seen in Figure 12 in which BER after channel correction (-) and without channel correction (.-) are shown.

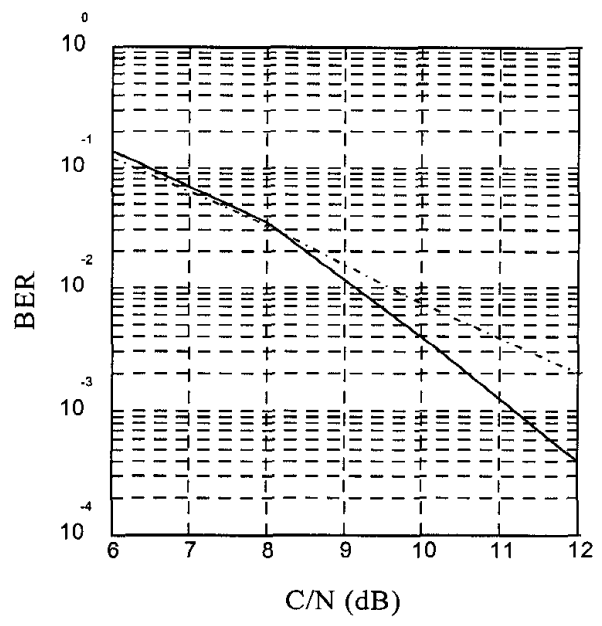

\section{Figure 12 BER of 16 QAM-OFDM (2K) after F1} channel

On the other hand, when the channel is more frequency-selective as P1 channel defined in [11], channel correction is always needed. Figure 13 shows the BER performance of a QPSK-OFDM (8K) system after this P1 channel: (1) and (2) have been obtained without correction and (3) and (4) with channel correction (without and with interleaving respectively).

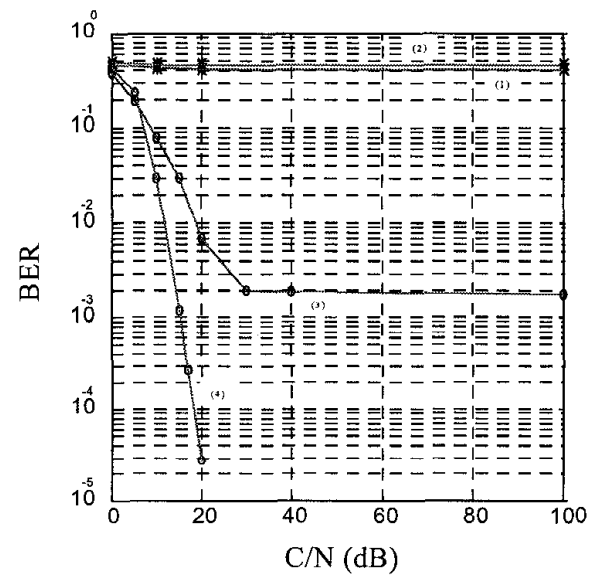

Figure 13 BER of QPSK-OFDM (8K) after P1 channel 


\subsection{Optimum power of pilot sub-carriers}

As mentioned before, some pilot sub-carriers are inserted in the OFDM frame for channel estimation purposes. The power of the pilots can be varied with respect to that of the information sub-carriers in order to reduce estimation errors. However a trade-off between $C / N$ ratio of information sub-carriers and channel estimation is required.

The parameter $P=\frac{\text { pot }_{\text {pilotos }}}{\text { pot }_{\text {resto }}}$ has been defined and its optimum value has been searched by simulation. Results for a QPSK-OFDM $2 \mathrm{~K}$ system are shown in Figure 14 leading to an optimum value of 2 .

The same value is obtained when the digital modulation and/or the number of sub-carriers are changed.

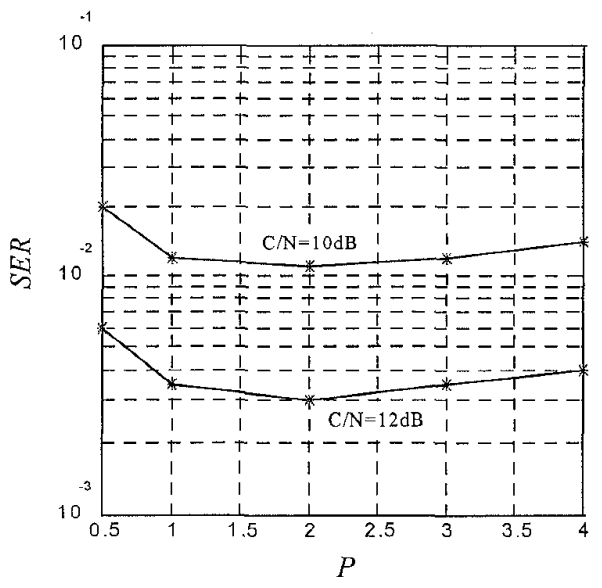

Figure 14 SER versus parameter $P$ in a QPSKOFDM 2K system

\section{ERROR PERFORMANCES}

DVB-T standard [1] defines many different transmission modes by selecting the inner code, digital modulation, Guard Time duration and number of subcarriers. For some of these combinations the $C / N$ ratios required to obtain a $\mathrm{BER}=2 \cdot 10^{-4}$ (this means Quasi Error Free performance after Reed-Solomon decoding) are provided in the standard specifications as indicative values that must be confirmed by testing. These values assume ideal channel estimation and synchronization in the presence of F1 and P1 channels [1].

With the aim of reducing the number of cases for study, the following modes have been considered in the $\mathrm{dTTb}$ project [2]:

- Mode 1: 64-QAM, 2/3 code.

- Mode 2: 16-QAM, $1 / 2$ code.

- Mode 3: 4-QAM (QPSK), 1/2 code.

- Mode 4: 64-QAM, 1/2 code.
- Mode 5: 16-QAM, 3/4 code.

- Mode 6: 64-QAM, $\alpha_{1}=2, \alpha_{2}=1$, two levels of hierarchy and $1 / 2$ code for HP, $3 / 4$ code for MPLP.

All of them consider $2 \mathrm{~K}$ and $8 \mathrm{~K}$ sub-carriers.

The $C / N$ values required to obtain this BER in nonideal conditions are presented here for Modes 2-5 and compared to those provided in [1]. Table 2 presents these values for a $2 \mathrm{~K}$ system.

Table $2 C / N(\mathrm{~dB})$ to achieve $\mathrm{BER}=2 \cdot 10^{-4}$ in 2K-OFDM

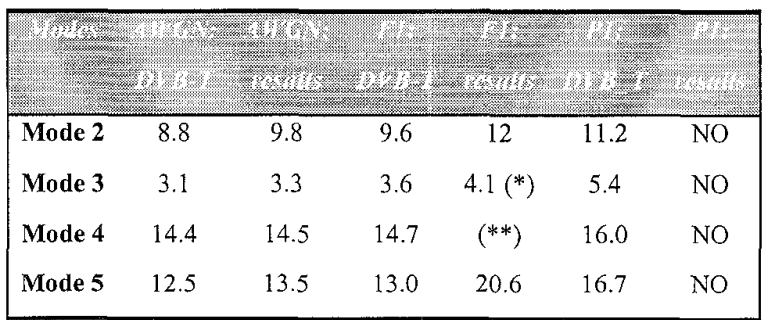

$\left.{ }^{*}\right)$ without channel correction

(**) saturation in $\mathrm{BER}=4 \cdot 10^{-4}$

The values for an $8 \mathrm{~K}$ system are shown in Table 3.

It should be noted that the $C / N$ values that are given in [1] are identical for $2 \mathrm{~K}$ and $8 \mathrm{~K}$ sub-carriers both in AWGN and multi-path channel conditions. This is due to the fact that ideal channel estimation has been considered in the standard. Obviously the situation is different when the channel is really estimated as shown in Tables 3 and 4.

Table $3 C / N(\mathrm{~dB})$ to achieve $\mathrm{BER}=2 \cdot 10^{-4}$ in 8K-OFDM

\begin{tabular}{|c|c|c|c|c|}
\hline Mode 2 & 8.8 & 9.8 & 9.6 & 11.4 \\
\hline Mode 3 & 3.1 & 3.3 & 3.6 & $4.2\left({ }^{*}\right)$ \\
\hline Mode 4 & 14.4 & 14.5 & 14.7 & 17.5 \\
\hline Mode 5 & 12.5 & 13.5 & 13.0 & 16.5 \\
\hline \multicolumn{5}{|c|}{ (*)without channel correction } \\
\hline & \multicolumn{4}{|c|}{$(* *)$ saturation in $\mathrm{BER}=5 \cdot 10^{-3}$} \\
\hline & \multicolumn{4}{|c|}{$(* * *)$ saturation in $\mathrm{BER}=4.5 \cdot 10^{-3}$} \\
\hline
\end{tabular}

On the other hand, it is not always convenient to correct the channel effects, as discussed in section 3.3. Values marked with $\left(^{*}\right)$ have been obtained without this correction. 
The $C / N$ values required in our prototype under AWGN conditions are very similar to those given in [1].

When the channel is $\mathrm{F} 1$ the difference between the $\mathrm{C} / \mathrm{N}$ values given in the standard and the obtained ones increases due to the non-ideal channel estimation. On the other hand, it wan be seen that channel effects are best corrected with $8 \mathrm{~K}$ sub-carriers.

Finally, if the channel is $\mathrm{P} 1$ the real obtained performance is much worse than expected in [1] for $8 \mathrm{~K}$ and it is not possible to achieve the desired BER values with $2 \mathrm{~K}$ because of the smaller number of pilot subcarriers.

These performance results have been obtained without using channel state information in the inner decoder. The required $C / N$ values could be lowered if this information was used. This fact is subject to further work.

\section{CONCLUSIONS}

The European Digital Television system for Terrestrial Broadcasting specified within the dTTb Project and the DVB-T standard is very complex, allowing a variety of configurations and operation modes. A parameter optimization process has been carried out in order to build a prototype containing the main elements considered this standard.

In order to achieve the best bit error rates, the following points have been studied:

- interleaving

- convolutional punctured codes and Viterbi decoding

- pilot-based channel correction

- optimum jower of pilot sub-carriers

Finally, a realistic BER performance has been provided and compared to the suggested ideal values given in the standard.

Although the results in the AWGN channel agree, the performance achieved in non-ideal conditions is poorer than expected in multi-path environments.

It should be noted that the use of channel state information in the inner decoder could lead to a better performance. However, this fact is subject to further work.

\section{REFERENCES}

[1] EBU/CENELEC/ETSI JTC, 'Digital broadcasting systems for television, sound and data services, framing structure, channel coding and modulation for digital terrestrial television', January 1996, TM 1545 rev. 2.

[2] dTTb Module 3, 'System Specification for the Second dTTb Demonstrator', Version 2.2, February 1996.
[3] H. Imai, S. Hirakawa, 'A new multilevel coding method using error correcting codes', IEEE Trans. on Information Theory, IT-23(3), pp: 371-377, May 1977.

[4] Y. Yasuda, K. Kashiki, Y. Hirata, 'High-Rate Punctured Convolutional Codes for Soft Decision Viterbi Decoding', IEEE Trans. on Communications, vol. COM32, no. 3, pp: 315-317, March 1984.

[5] Ana García Armada, Miguel Calvo, 'Rapid Prototyping of a Test Modem for Terrestrial Broadcasting of Digital Television', IEEE Trans. on Consumer Electronics, Vol. 43, no. 4, pp: 1100-1109, November 1997.

[6] Leonard J. Cimini, 'Analysis and Simulation of a Digital Mobile Channel Using Orthogonal Frequency Division Multiplexing', IEEE Trans. on Communications, Vol. COM-33, No. 7, pp. 665-675, July 1985.

[7] B. Le Floch, R. H. Lassalle, D. Castelain, 'Digital Sound Broadcasting to Mobile Receivers', IEEE Trans. on Consumer Electronics, Vol. 35, No. 3, pp: 493-503, August 1989.

[8] K. Jacobsen, J. Cioffi, 'An Efficient Digital Modulation Scheme for Multimedia Transmission on the Cable Television Network', 43rd Annual National Cable Television Association (NTCA) Convention and Exposition, New Orleans, LA, May 1994.

[9] S. B. Weinstein, P. M. Ebert, 'Data Transmission by Frequency-Division Multiplexing Using the Discrete Fourier Transform', IEEE Trans. on Communication Technology, Vol. COM-19. No. 5, pp: 628-634, October 1971.

[10] Ana García Armada, Miguel Calvo, Leandro de Haro: 'Diseño del Subsistema de Sincronismo para un sistema de comunicaciones basado en OFDM', Actas del XII Symposium Nacional de la URSI, pp: 161-164, Bilbao 1997.

[11] Ana García Armada, Miguel Calvo, Leandro de Haro: 'Influence of the Sub-carrier Spacing in the Performance of an OFDM Communication System', Proc. of HDTV'97, pp: C1-4, Montreux (Switzerland) 1997.

[12] A. R. Calderbank, 'Multilevel Codes and Multistage Decoding', IEEE Trans. on Communications, vol. 37 , no. 3, pp: $222-229$, March 1989

Ana García Armada was born in Santiago de Compostela, Spain, in 1970.

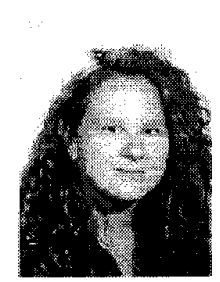

She received the Telecommunication Engineer degree from the ETSIT, Technical University of Madrid in July 1994 and the Ph.D. degree from the same University in 1998.

She has been involved in Simulation and Rapid Prototyping of the European Digital TV Terrestrial Broadcasting System (dTTb) based on COFDM signals. 
Beatriz Bardón was born in León, Spain, in 1970.

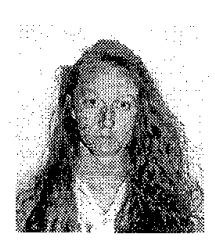

She received the Telecommunication Engineer degree from the ETSIT, Technical University of Madrid in February 1998.

She has been involved in simulation of the European Digital TV Terrestrial

Broadcasting System (dTTb) and the development of efficient simulation techniques.

Miguel Calvo was born in Pueyo de Jaca, Huesca, Spain in 1949.

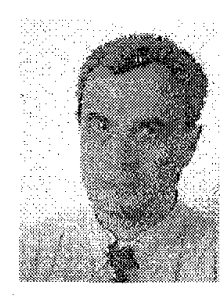

He got the Telecommunication Engineer degree from the ETSIT of the Technical University of Madrid in 1974 and the Ph.D. degree from the same University in 1979. He works as Professor in the Signals, Systems and Radiocommunications department.

Has co-authored a number of papers in technical reviews and contributed in a number of international conferences. He wrote a chapter in the book "Reflector and Lens Antennas. Analysis and Design using Personal Computers", C.J. Sletten editor, Artech House 1988. 